\title{
Coherence between self-reported and objectively measured physical activity in patients with chronic obstructive lung disease: a systematic review
}

This article was published in the following Dove Press journal:

International Journal of COPD

25 November 2016

Number of times this article has been viewed

\section{Mimi Thyregod ${ }^{1,2}$ \\ Uffe Bodtger ${ }^{1,2}$}

'Department of Respiratory Medicine, Naestved Hospital, Naestved, ${ }^{2}$ Institute for Regional Health Research, University of Southern Denmark, Odense, Denmark
Correspondence: Mimi Thyregod Department of Respiratory Medicine, Naestved Hospital, 6I Ringstedgade, DK 4700 Naestved, Denmark

Tel +4529828197

Email mimithyregod@gmail.com

\begin{abstract}
The beneficial effects of physical activity (PA) in patients with COPD, as well as the methods of their assessment, are well known and described. As objective measures of PA, such as the use of motion sensors, video recordings, exercise capacity testing, and indirect calorimetry, are not easily obtained in the daily clinical life, the reliability of the more accessible self-reported measurements of PA is important. In this review, we systematically identified original studies involving COPD patients and at least one parameter of self-reported and objective exercise testing, and analyzed every article for coherence between the objectively and self-reported measured PA. The studies are few, small, and very diverse, both in their use of questionnaires and objective measurements. Self-reported assessments were found to generally overestimate the level of PA compared to measurements made objectively by activity monitors; however, more studies are needed to rely solely on the use of PA questionnaires in COPD patients. The most accurate and valid questionnaires appear to be the self-completed Physical Activity Scale for the Elderly and the interviewer-completed Stanford Seven-Day Physical Activity Recall Questionnaire, but the ideal questionnaire still awaits construction. The motion sensors are accurate and validated in this patient group, especially SenseWear ${ }^{\mathrm{TM}}$, but not easily accessible in clinical practice, as they have various technical and adhesive difficulties.
\end{abstract}

Keywords: respiratory medicine, COPD, physical activity monitoring, questionnaire, motion sensor

\section{Introduction}

The level of PA in patients with COPD is inversely correlated with disease severity: lung function decline, disease burden, frequency of hospitalization, and mortality. ${ }^{1,2} \mathrm{PA}$ is defined as any bodily movement produced by skeletal muscles that results in energy expenditure. ${ }^{3}$ In COPD patients, the amount of PA is reduced compared to matched controls, as a result of - among other things - decreased threshold for exercise-induced dyspnea due to the irreversible airflow obstruction. ${ }^{4} \mathrm{PA}$ is efficacious in alleviating the vicious circle of deconditioning, and is the cornerstone of pulmonary rehabilitation. ${ }^{5,6}$

In COPD, correct assessment of PA in daily life is crucial to allocate patients to correct interventions: self-training, tele-health-based training, community-based or hospital-based pulmonary rehabilitation. In addition, knowledge of PA levels can motivate patients to improve, thereby reducing the morbidity and mortality by maintaining a proper PA level. ${ }^{7-9}$ Unfortunately, the correct assessment of a patient's PA levels is difficult, and no GOLD standard exists to quantify the amount of PA. ${ }^{10}$

PA levels can be assessed objectively or subjectively (self-reported), prospectively or retrospectively, and in experimental or real-life settings. Exercise capacity tests 
(objective/prospective/experimental setting), eg, the sixminute walk test (6MWT) or the incremental shuttle walk test (ISWT), are routinely used in rehabilitation programs and then a patient's daily PA levels are extrapolated from these tests, although a recent study showed a moderate-to-weak correlation to PA levels when the number of steps were measured by a pedometer. ${ }^{11}$ Other objective assessments often used in research settings or in athletes are the measurements of $\mathrm{VO}_{2}$ max and energy expenditure with indirect calorimetry (DLW method), which are considered as golden standard methods. ${ }^{12}$ Yet, assessment of PA energy expenditure does not quantify the duration, frequency, and intensity of the PA performed. Video recordings or activity monitors are examples of objective, prospective assessments in real-life settings (see "Objective methods for quantification of daily PA" section below), and the importance of the latter has recently been elaborately reviewed by Liao et al. ${ }^{13}$ Self-reported PA is more easily accessible and cheaper in both clinical life and epidemiological studies compared to video recordings or activity monitors - although often not very reliable. The most commonly used methods are self-reported, retrospective questionnaires on PA, whereas daily entries in a PA diary are less often used. Few questionnaires have been validated in COPD populations, which are characterized by much lower levels of PA compared to the general population (see "Objective methods for quantification of daily PA" section below).

Therefore, we aimed at performing a systematic review to investigate the agreement between self-reported and objectively measured PA in daily life, as well as the reliability and validity of each method and most importantly, the clinical application thereof. The following two sections describe the different methods followed for both subjective/self-reported and objective assessments.

\section{Objective methods for quantification of daily PA}

The Tuscan Renaissance artist and inventor Leonardo da Vinci drew the first sketches of a motion sensor in the 15th century: a pedometer held by both hands and looking like a wheelbarrow. ${ }^{14}$ Today, motion sensors are body-worn and divided into three categories: accelerometer (uniaxial, biaxial, triaxial), multisensor device, and pedometer. Accelerometers detect activity through changes in velocity as well as frequency, intensity, and duration of the activity. Their cost and technical complexity are higher than regular pedometers that only collect steps by vertical accelerations. The multisensor device combines the triaxial accelerometer with sensors (heat flux, galvanic skin response and skin temperature). Within each category, motion sensors differ in economic costs, technical complexity, and placement on the body (hip, arm, ankle, etc.). This is often a practical challenge in patients using walking aids, such as a walking stick or a walker, in addition to the use of motion systems in different environments such as in the water to record swimming or bicyling as examples. ${ }^{15}$

\section{Questionnaires for quantification of daily PA}

Self-reported measurements include diaries, logbooks, and questionnaires. Overall, questionnaires are classified into three categories: global, recall, and quantitative. ${ }^{16}$ The global questionnaire is short, self-administered, and usually consists of 2-4 questions, and can be used to assess whether a patient fulfills the international daily PA requirement of 30 minutes. The short recall questionnaire commonly includes 7-12 items and can be either interviewer- or selfadministered. The quantitative questionnaire is longer, typically includes 20-60 questions and provides in greater detail the PA level over a longer period of time.

The following validated questionnaires were used in the papers included in the study: PASE, PAR, Baecke Physical Activity Questionnaire, ZPAQ, and MARQ. ${ }^{17-19}$ Other validated questionnaires for the COPD population include Yale Physical Activity Survey ${ }^{20}$ and Minnesota Leisure Time Physical Activity Questionnaire, among others. ${ }^{20,21}$ A summary of the questionnaires featured in this review is presented in Table 1. The questionnaires are divided into four categories: multi- or single-item questionnaire, and validated or nonvalidated questionnaire in COPD populations.

\section{Methods}

A literature search was performed using PubMed on March 5, 2016, using the terms "Physical activity", "Monitoring" or "Measuring", "COPD", "Self-reported", “objective", and "correlation" or "coherence". We had no limitations concerning the publication year. Inclusion criteria were the following: 1) studies including patients with known COPD, 2) original studies involving PA, 3) objective measurement of the level of daily PA, 4) self-reported measurement of the level of daily PA, 5) peer-reviewed papers published in English. Exclusion criteria were the following: 1) studies including patients with diagnoses other than COPD, and without clear data separation between COPD and non-COPD groups and 2) studies reporting only subjective or objective PA measurement.

All identified abstracts were independently screened by two persons (MT and UB) and discussed with an experienced pulmonologist in COPD (Dr Anders Løkke) in case of disagreement. 
Table I Overview of the questionnaires

\begin{tabular}{|c|c|c|c|c|c|c|}
\hline Study & Questionnaire & $\begin{array}{l}\text { Administration } \\
\text { form }\end{array}$ & No of questions & $\begin{array}{l}\text { Recall } \\
\text { time }\end{array}$ & $\begin{array}{l}\text { Classification of } \\
\text { physical activity }\end{array}$ & Outcome \\
\hline 18 & PAR & Interviewer & $\begin{array}{l}4 \text { including complete } \\
\text { minute to minute diary }\end{array}$ & 7 days & Moderate, hard, very hard & Minutes \\
\hline 18 & Baecke & Self & 16 & 12 months & Light, moderate, hard & Minutes \\
\hline 18 & Zutphen & Self & 17 & I month & Moderate, hard, very hard & Minutes \\
\hline 17,18 & PASE & Self & 32 & 7 days & $\begin{array}{l}\text { Light, moderate, strenuous, } \\
\text { recreation }\end{array}$ & Hours $->$ score \\
\hline 17 & SBAS & Self & 2 & I day & $\begin{array}{l}\text { Inactive, light, moderate, hard, } \\
\text { very hard }\end{array}$ & Score \\
\hline 19 & MARQ & Self & - & - & All activities & Energy expenditure \\
\hline 23 & SQUASH & Self & 13 & 7 days & Light, moderate, intense & $\begin{array}{l}\text { Intensity scores based } \\
\text { on the reported effort }\end{array}$ \\
\hline
\end{tabular}

Abbreviations: MARQ, Modified Activity Recall Questionnaire; PAR, the Stanford Seven-Day Physical Activity Recall Questionnaire; PASE, Physical Activity Scale for the Elderly; SBAS, Stanford Brief Activity Scale; SQUASH, Short Questionnaire to Assess Health-enhancing physical activity.

\section{Important definitions}

SPI: the PAL is obtained by dividing the total daily energy expenditure by the resting energy expenditure, measured by an activity monitor. SPI is defined as PAL $<1.40 .{ }^{17}$

MET: a physiological measure expressing the energy cost of PA, as measured by an activity monitor. MET score is defined as follows: $<3$ light activities, 3-6 moderate activities, and $>6$ vigorous activities. ${ }^{18}$

\section{Outcome}

Primary outcome: difference in PA. Self-reported PA as a fraction of measured PA, possibly reported in the original study as Pearson's correlation.

Secondary outcomes: Percentage of SPI by questionnaire compared to objective measurements.

\section{Results}

The search process resulted in identification of 39 abstracts, and after review, 7 original papers (Figure 1) were selected for the study. During the review process, most studies were discarded due to a lack of either an objective or a subjective measure of self-reported PA ( $\mathrm{n}=30)$. A few studies were published in non-English languages $(\mathrm{n}=2)$. Table 2 depicts differences in sample size, PA and methods for objective and subjective measurements of PA in the various studies. All included papers involved motion sensors. The study could not find any relevant RCTs on the subject, only cohort studies.

\section{Risk of bias}

According to the NOS, we assessed the quality of the included studies, as given in Table $3 .^{22}$ Agreement between the reviewers (MT and UB) was calculated and expressed as a percentage and quantified by kappa statistics; the results revealed an agreement of $81 \%$ and a kappa value of 0.74 .

\section{Validated multi-item questionnaire}

In an English study, PAR was compared with a SenseWearTM (Bodymedia, Pittsburgh, PA, USA) multisensor and validated using three other validated PA questionnaires: Baecke, PASE, and ZPAQ (Table 2). ${ }^{13,18}$

The study included 43 COPD patients who wore SenseWearTM for 7 days. A significant correlation between
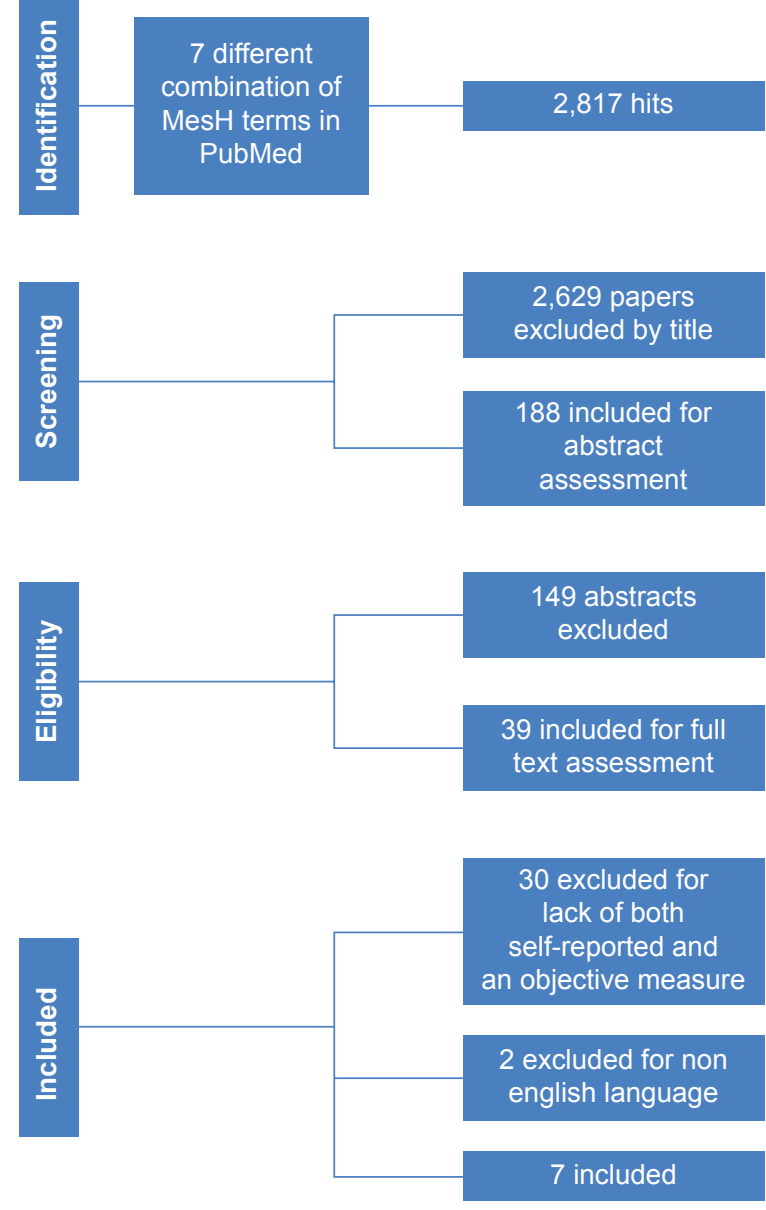

Figure I Flow diagram of search results. 


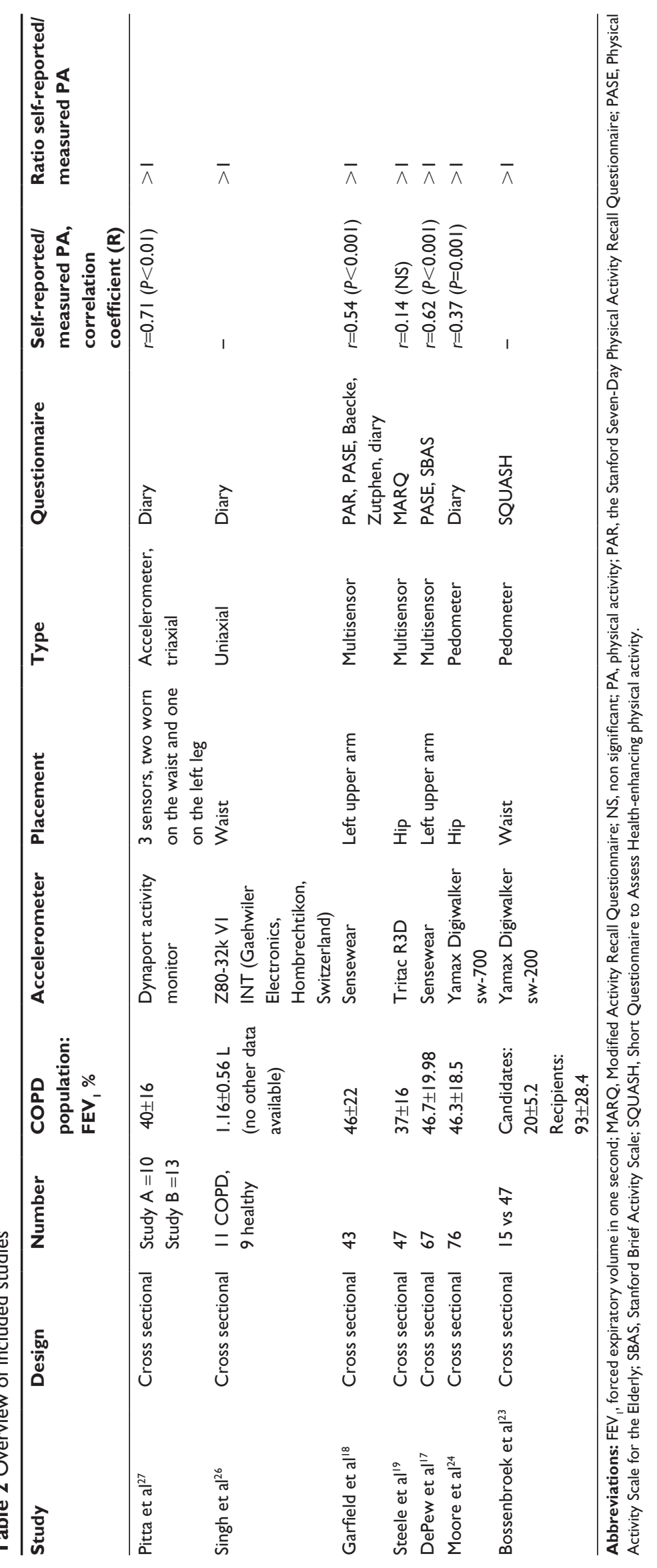


Table 3 Risk of bias

The Newcastle-Ottawa Scale (NOS) for assessing the quality of nonrandomized studies

\begin{tabular}{|c|c|c|c|c|}
\hline \multirow[t]{2}{*}{ Study } & \multicolumn{4}{|c|}{ Risk of bias assessment } \\
\hline & $\begin{array}{l}\text { Selection } \\
(0-3)\end{array}$ & $\begin{array}{l}\text { Comparability } \\
(0-2)\end{array}$ & $\begin{array}{l}\text { Outcome } \\
(0-3)\end{array}$ & $\begin{array}{l}\text { Total } \\
\text { score }\end{array}$ \\
\hline Garfield et al ${ }^{18}$ & 2 & - & 3 & 5 \\
\hline DePew et al ${ }^{17}$ & 2 & - & 3 & 5 \\
\hline Steele et a ${ }^{19}$ & 2 & - & 3 & 5 \\
\hline Bossenbroek et $\mathrm{al}^{23}$ & I & - & 3 & 4 \\
\hline Moore et $\mathrm{a}^{24}$ & 0 & - & 3 & 3 \\
\hline Singh and Morgan ${ }^{26}$ & 2 & 1 & 2 & 5 \\
\hline Pitta et $\mathrm{al}^{27}$ & 2 & - & 3 & 5 \\
\hline Mean & 1.6 & 1 & 2.9 & 32 \\
\hline
\end{tabular}

Notes: Domain I (selection) scored out of 3 , and not out of 4, as the "outcome of interest was not present at start of the study", was considered not appropriate for this review.

the TEE derived by PAR and SenseWear ${ }^{\mathrm{TM}}$ was observed in patients with moderate PA (MET $\geq 3, r=0.83$ ). Furthermore, PAR correctly predicted patients at each end of the PA scale as very inactive and very active patients. In addition, the patients completed a 6MWT that did not predict the active patients, according to the PA detected by SenseWear ${ }^{\mathrm{TM}}$.

Likewise, DePew et al applied both the PASE questionnaire and SenseWear ${ }^{\mathrm{TM}} .{ }^{17}$ PASE demonstrated a moderate correlation with the PAL measured by SenseWear ${ }^{\mathrm{TM}}$ in 67 COPD patients with a positive correlation coefficient $(r=0.62)$. Using a cut-off PASE score of $<111$, it predicted SPI measured by SenseWear ${ }^{\mathrm{TM}}$, with a negative predictive value of 0.65 and a positive predictive value of 0.83 , indicating an underestimation of SPI utilizing a PASE questionnaire.

The best predictor of SPI was the $\mathrm{mMRC} \geq 3$, although PASE and mMRC together achieved an even better correlation (concordance correlation coefficient 0.833 ).

Another study compared the MARQ, a self-reported PA questionnaire which assesses energy expenditure, with the TriTrac-R3D accelerometer in 47 COPD patients. ${ }^{19}$

No significant relationship was found between reported and measured PA, and the low correlation $(r=0.4)$ suggests profound overestimation of PA measured by the MARQ. In the same study, PA observed during 6MWT correlated significantly with that measured by TriTrac-R3D $(r=0.74)$.

\section{Nonvalidated multi-item questionnaire}

A Dutch study investigated PA in 47 lung-transplant candidates and 15 lung-transplant recipients. Measurement with a pedometer (with a wearing time of 10 days, where the last 7 days were included in the measurements) was compared to the SQUASH. ${ }^{23}$ The correlation coefficient was not calculated between the pedometer and the SQUASH. The SQUASH divides the activities in MET scores. In the study, the total amount of activity was calculated and defined as "steps equivalent", using the data from the pedometer (steps) and the SQUASH, converting PA where steps could not be counted, eg, cycling and swimming. The calculated steps equivalent per day was almost twice as high in the lung-transplant candidate group when compared with the steps measured by the

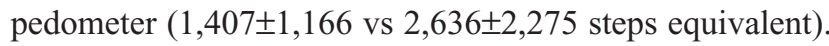
In the lung-transplant recipient group, the calculated steps equivalent was almost one-third higher than the pedometer-measured steps $(6,642 \pm 2,886$ vs $9,038 \pm 4,583$ steps equivalent). Even though the correlation coefficient was not calculated, the study showed a relative overestimation of the self-reported PA compared to the measured PA.

\section{Nonvalidated single-item score}

A similar study was carried out by Moore et al in 76 COPD patients using a pedometer and a simple 7-day PA diary. ${ }^{24}$ A significant, but poor or moderate correlation $(r=0.37)$ was demonstrated using a modified diary developed by Follick et al. ${ }^{25}$ It consisted of four categories of activities (sleeping, sitting, standing/walking, lying) and was completed a minimum of three times per day. The authors concluded that the pedometer used underestimated PA levels, and that the diary was a more accurate measure of PA, yet no other objective measure was established to confirm this assertion. A small study with 11 COPD patients and 9 healthy age-matched controls investigated if brisk walking was detected by an accelerometer worn for 48 hours. ${ }^{26}$ The COPD patients had recently completed pulmonary rehabilitation, and brisk walking was defined as $60 \%$ of the predicted peak oxygen consumption in ISWT. No data on how brisk walking was defined in the healthy age-matched controls were provided. The COPD patients recorded daily PA in 10 different categories: lying, sitting, sitting activity, standing, standing activity, personal, walking (brisk), walking (slow/ intermittent), driving, and others. There was a significant correlation between the diary recorded brisk walking and that counted by the accelerometer, but there was a wide range in the standard deviation scores in all other categories. No results on whether the diary over- or underestimated PA according to the accelerometer were provided in the paper, but step count differed insignificantly between lying and all activities, except for brisk walking - suggesting overestimation of self-reported PA.

Pitta et al performed two unrelated studies: A and B. ${ }^{27}$ In study A, a small group of 10 patients with COPD correlated 
patient estimations, DAM, and video recordings in a 1-hour standardized protocol, whereas study B, consisting of 13 other COPD patients, correlated DAM, and a self-report in a regular-day protocol. The self-report consisted of a logbook, reporting on an hourly basis the time spent on each activity or body position. In study A, patients significantly underestimated sitting time in the self-report. DAM and video recordings were correlated, even though the DAM systematically slightly overestimated walking and cycling time. There was a high disagreement between the self-report and the video recordings. In study $\mathrm{B}$, patients significantly overestimated walking time $(r=0.71)$ and underestimated standing time in the self-report compared to the DAM.

\section{Discussion}

The review unveils the existence of few and heterogeneous studies using accelerometers and self-reported PA simultaneously, resulting in a low validity and test-retest reliability. Heterogeneity was observed in patient selection (ranging from mild to very severe COPD), intensity, and duration of PA. Steele et al confirmed the correlation between $\mathrm{FEV}_{1}$ and PA, as measured by the accelerometer and the 6MWT, highlighting the relationship between airway obstruction and maximum exercise capacity. ${ }^{19,23,28}$ A general overestimation of self-reported PA was demonstrated, and no reliable PA questionnaire was found. ${ }^{17,19,27,29}$ The most accurate tool for self-reported PA based on the papers assessed here is to keep a daily diary with a description of all activities. ${ }^{24}$

However, two questionnaires predicted very low levels of daily PA: PASE and PAR. The PASE questionnaire was able to predict SPI in COPD patients, although, together with mMRC, it had a more accurate outcome. ${ }^{17}$ The PAR questionnaire was the only questionnaire to reach significant correlation with SenseWear ${ }^{\mathrm{TM}} .{ }^{18}$ However, this finding was only observed in the subgroup of COPD patients reaching a moderate PA level (MET $\geq 3$ ). The authors concluded that PAR predicted PA only as a binary outcome (active/inactive) with respect to PA levels. Since PAR was an interviewer-led questionnaire, it was difficult to use this in an epidemiological setting.

Various challenges are associated with PA recollection in COPD patients. An accurate PA questionnaire in the, mostly sedentary, COPD population should include various questions on the reasons for low PA. The PASE questionnaire was designed for an elderly population, whereas the validation of the SQUASH questionnaire was done in a subgroup including mostly men (70\%), with a mean age of 44 years, and $50 \%$ had a good or high fitness level, which is far from that expected for the COPD population..$^{17,30}$
Questionnaires are either interviewer administered or self-completed by the patient. Of the questionnaires included in this review, only PAR was interviewer administered, with the risk of over-administration the answers. On the other hand, there might be a risk of missing data when using selfadministered questionnaires, as well as misunderstanding of both content- and time-related questions.

Cognitive impairment in COPD has gained increasing attention. ${ }^{31}$ Recollecting the details of daily life activities, including PA, is evidently hampered in the most diseased COPD patients, and therefore reliability of the data collected has been questioned. ${ }^{27,32,33}$ The health benefits of normal PA are widely accepted. ${ }^{5,6,13}$ These influence the patients' selfreporting measurements, resulting in a tendency to overesti-

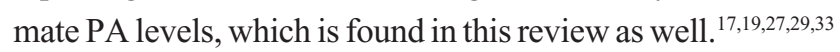
Recall bias is also affected by time factors, such as when and how the questionnaire is to be completed, and the amount of time the patient has to recall. The Baecke questionnaire covers the preceding year, and previously we have shown that otherwise healthy patients with seasonal pollen allergy only recollected the single day with the most symptoms 4 months after the pollen season. Thus, recollection of PA during an entire year is unlikely to be reliable or correct. ${ }^{17,34}$

A significant dropout rate is also a well-known problem in the COPD population, making it even harder to generalize the results to the entire COPD population as only the healthiest patients complete each study. ${ }^{28}$

Accelerometers have very different ways of calculating and reporting energy expenditure and PA, and it is mandatory to convert the reported output for comparison with that of questionnaire-based self-reported PA, work capacity testing, or other activity monitors. A device's test-retest reliability and validity is typically derived in a non-COPD population. Measuring PA in the relatively inactive COPD population results in a rather narrow PA range as most time is spent sedentary with a low PA level concerning both duration and intensity. ${ }^{12}$ This results in a demand for highly sensitive activity monitors that can detect differences in PA even in very inactive patients, including a measurement of PA that does not involve total body movement, such as weight lifting and stationary cycling. ${ }^{19,29}$

In addition to the challenge of recollecting PA, Pitta et al reported adherence difficulties upon using an accelerometer in 19\% of COPD subjects including body placement and technical issues, such as battery problems. ${ }^{29}$ Additionally, Moore et al demonstrated that $18 \%$ of COPD patients failed to use a simple pedometer correctly; furthermore, $5 \%$ completed the diary insufficiently. ${ }^{24}$ These failure rates are probably higher 
in unselected, everyday patients who do not feel obliged to please an investigator during a clinical trial.

The limitations of this study are the lack of high-quality, high-volume studies and not all the included studies published coherence between self-reported and objectively measured PA. Only two studies included $>50$ patients. ${ }^{17,24}$ This review unveils a critical need for a more accurate PA questionnaire in the COPD population, as objective measures are costly, time-consuming and prone to adherence difficulties as well as patient-related issues.

\section{Conclusion}

The PA level has an impact on both prognosis of the patient and the disease severity. Assessment of daily PA is warranted to optimize PA-directed intervention. In the surprisingly few and small studies available on measurements of PA in daily life, self-reported assessments generally overestimated the level of PA measured objectively by activity monitors. However, the level of evidence is hampered by significant inter-study heterogeneity precluding statistical evaluation by meta-analysis. The most accurate and valid questionnaires appear to be the self-completed PASE and the interviewer-completed PAR questionnaires, but the ideal questionnaire still awaits construction. Establishment of a well-defined golden standard of daily-life PA will improve the efforts in the development of a simple, accurate, and valid measurement of daily-life PA.

\section{Abbreviations}

PA, physical activity; COPD, chronic obstructive pulmonary disease; 6MWT, six-minute walking test; ISWT, incremental shuttle walk test; $\mathrm{VO}_{2}$ max, maximum oxygen consumption; DLW, double labeled water; PASE, Physical Activity Scale for the Elderly; PAR, the Stanford Seven-Day Physical Activity Recall Questionnaire; ZPAQ, Zutphen Physical Activity Questionnaire; MET, metabolic equivalent of task; MARQ, Modified Activity Recall Questionnaire; SPI, severe physical inactivity; TEE, total energy expenditure; PAL, physical activity level; NOS, Newcastle-Ottawa Scale; SQUASH, Short QUestionnaire to ASsess Health-enhancing physical activity; DAM, DynaPort Activity Monitor; $\mathrm{FEV}_{1}$, forced expiratory volume in one second; GOLD, Global Initiative for Chronic Obstructive Lung Disease; mMRC, Modified Medical Research Council Dyspnea Scale; RCT, randomized controlled trial.

\section{Acknowledgment}

The authors gratefully thank Anders Løkke, MD, Department of Respiratory Medicine, Aarhus University Hospital,
Denmark, for agreeing to settle possible disagreement between the authors during the review process.

\section{Disclosure}

The authors report no conflicts of interest in this work.

\section{References}

1. Cindy Ng LW, Mackney J, Jenkins S, Hill K. Does exercise training change physical activity in people with COPD? A systematic review and meta-analysis. Chron Respir Dis. 2012;9(1):17-26.

2. Kanda M, Minakata Y, Matsunaga K, et al. Validation of the triaxial accelerometer for the evaluation of physical activity in Japanese patients with COPD. Intern Med. 2012;51(4):369-375.

3. Caspersen CJ, Powell KE, Christenson GM. Physical activity, exercise, and physical fitness: definitions and distinctions for health-related research. Public Health Rep. 1985;100(2):126-131.

4. Wilson JJ, O'Neill B, Collins EG, Bradley J. Interventions to increase physical activity in patients with COPD: a comprehensive review. COPD. 2015;12(3):332-343.

5. McCarthy B, Casey D, Devane D, Murphy K, Murphy E, Lacasse Y. Pulmonary rehabilitation for chronic obstructive pulmonary disease. Cochrane Database Syst Rev. 2015;(2):CD003793.

6. Rugbjerg M, Iepsen UW, Jorgensen KJ, Lange P. Effectiveness of pulmonary rehabilitation in COPD with mild symptoms: a systematic review with meta-analyses. Int J Chron Obstruct Pulmon Dis. 2015;10: 791-801.

7. Zanaboni P, Hoaas H, Aaroen Lien L, Hjalmarsen A, Wootton R. Longterm exercise maintenance in COPD via telerehabilitation: a two-year pilot study. J Telemed Telecare. Epub 2016 Feb 16.

8. Mantoani LC, Rubio N, McKinstry B, MacNee W, Rabinovich RA. Interventions to modify physical activity in patients with COPD: a systematic review. Eur Respir J. 2016;48(1):69-81.

9. Haskell WL, Lee IM, Pate RR, et al. Physical activity and public health: updated recommendation for adults from the American College of Sports Medicine and the American Heart Association. Med Sci Sports Exerc. 2007;39(8):1423-1434.

10. Dyrstad SM, Hansen BH, Holme IM, Anderssen SA. Comparison of self-reported versus accelerometer-measured physical activity. Med Sci Sports Exerc. 2014;46(1):99-106.

11. Zwerink M, van der Palen J, van der Valk P, Brusse-Keizer M, Effing T. Relationship between daily physical activity and exercise capacity in patients with COPD. Respir Med. 2013;107(2):242-248.

12. Rabinovich RA, Louvaris Z, Raste Y, et al; PROactive Consortium. Validity of physical activity monitors during daily life in patients with COPD. Eur Respir J. 2013;42(5):1205-1215.

13. Liao S-Y, Benzo R, Ries AL, Soler X. Physical activity monitoring in patients with chronic obstructive pulmonary disease. JCOPD F. 2014; 1(2):155-165.

14. MacCurdy E. The Notebooks of Leonardo Da Vinci. New York: Reynal \& Hitchcock; 1938.

15. Andersson M, Janson C, Emtner M. Accuracy of three activity monitors in patients with chronic obstructive pulmonary disease: a comparison with video recordings. COPD. 2014;11(5):560-567.

16. Strath SJ, Kaminsky LA, Ainsworth BE, et al; American Heart Association Physical Activity Committee of the Council on Lifestyle and Cardiometabolic Health and Cardiovascular, Exercise, Cardiac Rehabilition and Prevention Committee of the Council on Clinical Cardiology, and Council. Guide to the assessment of physical activity: clinical and research applications: a scientific statement from the American Heart Association. Circulation. 2013;128(20):2259-2279.

17. DePew ZS, Garofoli AC, Novotny PJ, Benzo RP. Screening for severe physical inactivity in chronic obstructive pulmonary disease: the value of simple measures and the validation of two physical activity questionnaires. Chron Respir Dis. 2013;10(1):19-27. 
18. Garfield BE, Canavan JL, Smith CJ, et al. Stanford Seven-Day Physical Activity Recall questionnaire in COPD. Eur Respir J. 2012;40(2): 356-362.

19. Steele BG, Holt L, Belza B, Ferris S, Lakshminaryan S, Buchner DM. Quantitating physical activity in COPD using a triaxial accelerometer. Chest. 2000;117(5):1359-1367.

20. Donaire-Gonzalez D, Gimeno-Santos E, Serra I, et al; en representacion del PAC-COPD Study Group. Validación del cuestionario de actividad física de Yale en pacientes con enfermedad pulmonar obstructiva crónica [Validation of the Yale Physical Activity Survey in chronic obstructive pulmonary disease patients]. Arch Bronconeumol. 2011;47(11):552-560. Spanish.

21. Marin Royo M, Pellicer Ciscar C, Gonzalez Villaescusa C, et al; EPOC de la Sociedad Valenciana de Neumología (SVN). Actividad física y su relación con el estado de salud en pacientes EPOC estables [Physical activity and its relationship with the state of health of stable COPD patients]. Arch Bronconeumol. 2011;47(7):335-342. Spanish.

22. Wells GA, Shea B, O'Connell D, et al. The Newcastle-Ottawa Scale (NOS) for assessing the quality of nonrandomised studies in metaanalyses. Available from: http://www.ohri.ca/programs/clinical_epidemiology/oxford.asp. Accessed October 20, 2016.

23. Bossenbroek L, ten Hacken NH, van der Bij W, Verschuuren EA, Koeter GH, de Greef MH. Cross-sectional assessment of daily physical activity in chronic obstructive pulmonary disease lung transplant patients. J Heart Lung Transplant. 2009;28(2):149-155.

24. Moore R, Berlowitz D, Denehy L, Jackson B, McDonald CF. Comparison of pedometer and activity diary for measurement of physical activity in chronic obstructive pulmonary disease. J Cardiopulm Rehabil Prev. 2009;29(1):57-61.

25. Follick MJ, Ahern DK, Laser-Wolston N. Evaluation of a daily activity diary for chronic pain patients. Pain. 1984;19(4):373-382.
26. Singh S, Morgan MD. Activity monitors can detect brisk walking in patients with chronic obstructive pulmonary disease. J Cardiopulm Rehabil. 2001;21(3):143-148.

27. Pitta F, Troosters T, Spruit MA, Decramer M, Gosselink R. Activity monitoring for assessment of physical activities in daily life in patients with chronic obstructive pulmonary disease. Arch Phys Med Rehabil. 2005; 86(10):1979-1985

28. Frisk B, Espehaug B, Hardie JA, et al. Physical activity and longitudinal change in 6-min walk distance in COPD patients. Respir Med. 2014; 108(1):86-94.

29. Pitta F, Troosters T, Probst VS, Spruit MA, Decramer M, Gosselink R. Quantifying physical activity in daily life with questionnaires and motion sensors in COPD. Eur Respir J. 2006;27(5):1040-1055.

30. Wendel-Vos GC, Schuit AJ, Saris WH, Kromhout D. Reproducibility and relative validity of the short questionnaire to assess health-enhancing physical activity. J Clin Epidemiol. 2003;56(12):1163-1169.

31. Roncero C, Campuzano AI, Quintano JA, Molina J, Perez J, Miravitlles M. Cognitive status among patients with chronic obstructive pulmonary disease. Int J Chron Obstruct Pulmon Dis. 2016;11:543-551.

32. Antonelli Incalzi R, Marra C, Giordano A, et al. Cognitive impairment in chronic obstructive pulmonary disease - a neuropsychological and spect study. J Neurol. 2003;250(3):325-332.

33. Shephard R, Vuillemin A. Limits to the measurement of habitual physical activity by questionnaires. Br J Sports Med. 2003;37(3):197-206; discussion 206.

34. Bodtger U, Poulsen LK, Malling HJ. Retrospective assessment of seasonal allergic symptoms: over-rating but useful. Clin Exp Allergy. 2003;33(4):496-500.
International Journal of COPD

\section{Publish your work in this journal}

The International Journal of COPD is an international, peer-reviewed journal of therapeutics and pharmacology focusing on concise rapid reporting of clinical studies and reviews in COPD. Special focus is given to the pathophysiological processes underlying the disease, intervention programs, patient focused education, and self management protocols.

\section{Dovepress}

This journal is indexed on PubMed Central, MedLine and CAS. The manuscript management system is completely online and includes a very quick and fair peer-review system, which is all easy to use. Visit http://www.dovepress.com/testimonials.php to read real quotes from published authors. 